\title{
Prevalência do uso de substâncias psicoativas em gestantes e puérperas
}

Prevalence of psychoactive substance use in pregnant and puerperal women

Prevalencia del consumo de sustancias psicoactivas en mujeres embarazadas y puérperas

\section{Karen Barcelos Lopes ${ }^{\mathrm{I}}$, Juliane Portella Ribeiro ${ }^{\mathrm{II}}$, Alitéia Santiago Dilélio ${ }^{\mathrm{III}}$, Amanda do Rosário Tavares ${ }^{\mathrm{IV}}$, Beatriz Franchiniv ${ }^{\mathrm{V}}$, Melissa Hartmann ${ }^{\mathrm{VI}}$}

\begin{abstract}
Resumo: Objetivo: investigar a prevalência do uso de substâncias psicoativas em gestantes e puérperas atendidas no ambulatório de um Hospital Escola. Método: pesquisa transversal, descritiva, com amostra de 174 gestantes e puérperas. $\mathrm{Na}$ coleta de dados, utilizou-se o instrumento padronizado Alcohol, Smoking and Substance Involvement Screening Test (ASSIST) e um questionário sociodemográfico e gineco-obstétrico. A análise de dados foi realizada por meio da estatística descritiva. Resultados: a prevalência de uso de substâncias psicoativas entre as gestantes foi de 28,7 \% e 1,1\% entre as puérperas, com destaque para o álcool e tabaco. O tabaco constitui a substância com maior frequência de uso; que desencadeia maior desejo/urgência de consumo; maior taxa de tentativas de diminuição. Conclusão: o uso de substâncias psicoativas na gestação e puerpério constitui-se um desafio à saúde pública e para os profissionais de saúde, suscitando o desenvolvimento de ações educativas, bem como a captação precoce dessas mulheres.
\end{abstract}

Descritores: Enfermagem; Gravidez de Alto Risco; Período Pós-parto; Usuários de Drogas; Serviços de Saúde Maternoinfantil

Abstract: Objective: to investigate the prevalence of psychoactive substance use in pregnant and puerperal women attended at an outpatient clinic in a teaching hospital. Method: A descriptive transversal research with a sample of 174 pregnant and puerperal women. The Alcohol, Smoking and Substance Involvement Screening Test (ASSIST) and a sociodemographic and obstetric-gynecologic survey were utilized in data collection. Data analysis was performed through descriptive statistics. Results: the prevalence of psychoactive substance use among

\footnotetext{
I Enfermeira. Faculdade de Enfermagem. Universidade Federal de Pelotas, Pelotas, Rio Grande do Sul, Brasil. E-mail: karenbarcelos1@hotmail.com. Orcid: https://orcid.org/0000-0003-0734-938X

II Professora Adjunta. Doutora em Enfermagem. Faculdade de Enfermagem. Universidade Federal de Pelotas. Pelotas, Rio Grande do Sul, Brasil. E-mail: ju_ribeiro1985@hotmail.com. Orcid: https://orcid.org/0000-0002-1882-6762

III Professora Adjunta. Doutora em Epidemiologia. Faculdade de Enfermagem. Universidade Federal de Pelotas. Pelotas, Rio Grande do Sul, Brasil. E-mail: aliteia@gmail.com. Orcid: https://orcid.org/0000-0001-6718-2038

IV Acadêmica de Enfermagem. Faculdade de Enfermagem. Universidade Federal de Pelotas, Pelotas, Rio Grande do Sul, Brasil. E-mail: arosariotavares@icloud.com. Orcid: https://orcid.org/0000-0002-4262-1409

V Professora Adjunta. Doutora em Enfermagem. Faculdade de Enfermagem. Universidade Federal de Pelotas. Pelotas, Rio Grande do Sul, Brasil. E-mail: beatrizfranchini@hotmail.com. Orcid: https://orcid.org/0000-0003-3122-7091

VI Acadêmica de Enfermagem. Faculdade de Enfermagem. Universidade Federal de Pelotas, Pelotas, Rio Grande do Sul, Brasil. E-mail: hmelissahartmann@gmail.com. Orcid: https://orcid.org/0000-0002-3955-0558
} 
pregnant women was of $28.7 \%$ and $1.1 \%$ among puerperal women, with alcohol and tobacco as highlights. Tobacco constitutes the substance with greater frequency of use, triggering greater desire/urgency of consummation and a higher rate of intake lowering attempts. Conclusion: the use of psychoactive substances among pregnant and puerperal women constitutes a challenge to public health and for health professionals, eliciting the development of educational actions as well as early identification of these women.

Descriptors: Nursing; Pregnancy, high-risk; Postpartum period; Drug users; Maternal-child health services

Resumen: Objetivo: investigar la prevalencia del uso de sustancias psicoactivas en mujeres embarazadas y puérperas atendidas en el ambulatorio de un Hospital Escuela. Método: investigación transversal, descriptiva, con una muestra de 174 mujeres embarazadas y puérperas. En la recopilación de datos, se utilizó el instrumento estandarizadoAlcohol, Smoking and Substance Involvement Screening Test (ASSIST) y un cuestionario sociodemográfico y gineco-obstétrico. El análisis de los datos se realizó mediante estadísticas descriptivas. Resultados: la prevalencia del consumo de sustancias psicoactivas entre las embarazadas fue del $28,7 \%$, mientras que entre las puérperas fuel del 1,1\%, con destaque para el alcohol y el tabaco. El tabaco constituye la sustancia de mayor frecuencia de uso; que desencadena mayor deseo/urgencia de consumo; mayor tasa de tentativas de disminución. Conclusión: el uso de sustancias psicoactivas en elembarazo y el puerperio constituye un reto para la salud pública y para los profesionales de la salud, suscitando el desarrollo de acciones educativas, así como la captación temprana de estas mujeres.

Descriptores: Enfermería; Embarazo de alto riesgo; Periodo posparto; Consumidores de drogas; Servicios de salud materno-infantil

\section{Introdução}

O aumento progressivo de uso de substâncias psicoativas por mulheres no período gravídico-puerperal tem configurado um importante problema de saúde pública. A United States Substance Abuse and Mental Health, maior fonte de informação do Reino Unido, constatou que, no ano de 2018, 5,4\% das mulheres relataram usar drogas ilícitas durante a gravidez, observando um aumento substancial quando comparado com a 2010 , com $4,4 \%{ }^{1}$

Em estudo nacional sobre o uso de Crack no Brasil, realizado pela Secretária Nacional de Políticas sobre Drogas (SENAD) e a Fundação Oswaldo Cruz (FIOCRUZ) no ano de 2012, com uma amostra de 7.381 usuários, o sexo masculino foi predominante, no entanto, ressalta-se que $21,32 \%$ da amostra foram mulheres. Destas, aproximadamente $13 \%$ responderam que estavam grávidas no momento da entrevista. ${ }^{2}$ 
Estudo realizado no Maranhão, com uma amostra de 1.447 gestantes, identificou que 1,45\% fez uso de substâncias ilícitas (canabinoides e derivados e/ou cocaína e derivado), 22,32\% de bebidas alcoólicas e 4,22\% utilizavam cigarro. ${ }^{3}$ Em relação ao período puerperal, estudo de coorte, realizado em Campinas, no estado de São Paulo, com 674 mulheres, constatou que cerca de $25 \%$ declararam uso após o parto, sendo que mais de $5 \%$ faziam uso concomitantemente. A substância mais utilizada foi o álcool (20,6\%), seguido pelo tabaco (13,2\%), cocaína (2,3\%) e maconha e outras drogas $(1,9 \%){ }^{4}$

Destaca-se que, quando o uso envolve gestantes e puérperas, suas consequências possuem maior extensão, visto que pode ocorrer danos irreversíveis à mãe e ao feto. ${ }^{5} \mathrm{O}$ uso abusivo de álcool pode ocasionar alterações no organismo como doenças cardiovasculares, neoplasias, depressão e distúrbios neurológicos, além de dificuldade para ganho de peso gestacional, menor adesão às consultas de pré-natal e possível aumento no uso de outras substâncias. ${ }^{6}$ Estudo realizado no Irã, com 267 gestantes, apontou que o uso de crack está associado à maior taxa de pré-eclâmpsia, deslocamento prematuro da placenta, trabalho de parto prematuro e baixo peso ao nascer. ${ }^{7}$

Constata-se que as complicações do uso não se restringem apenas às gestantes ou puérperas, mas também ao feto e ao neonato. Pesquisadores indicam que a ocorrência de restrição de crescimento intrauterino, prematuridade, óbito fetal, síndrome de abstinência neonatal e morte súbita infantil são apenas alguns dos problemas relacionados ao uso na gestação. ${ }^{8-9}$ Além disso, o uso requer cuidados extras também no pós-parto, pois a puérpera pode ter crises de abstinência, agressividade, isolamento, dificuldade no convívio social, no autocuidado ou no cuidado do recém-nascido. ${ }^{10}$

Nesse contexto, a identificação precoce favorece a intervenção e cria possibilidade de acesso a serviços especializados de tratamento e alternativas de enfrentamento ao uso de substâncias psicoativas na gestação e puerpério, evitando e/ou amenizando complicações 
maternas e neonatais. ${ }^{11}$ No entanto, a detecção precoce do uso de substâncias psicoativas é apontada como uma lacuna na assistência à mulher no período gravídico-puerperal. Estudo com 25 gestantes atendidas em unidades Básicas de Saúde em Maringá, no estado do Paraná, evidenciou que os profissionais de saúde, de forma geral, não abordavam questões relacionadas ao uso de drogas, lícitas ou ilícitas, durante o acompanhamento pré-natal. ${ }^{12}$

A abordagem acerca do uso de substâncias, lícitas ou ilícitas, deve fazer parte da rotina de pré-natal, explorando a história pregressa e atual do consumo, quantidade e frequência. ${ }^{13}$ Reconhecendo as fragilidades existentes na atuação dos profissionais de saúde no rastreio e assistência às gestantes e puérperas usuárias de substâncias, pesquisadores apontam a necessidade de desenvolvimento de atividades de educação permanente para qualificar a captação precoce e ofertar acolhimento sensível às demandas e especificidades de saúde dessas mulheres. ${ }^{14}$

Diante do exposto, há necessidade de investigar o uso de substâncias psicoativas por gestantes e puérperas, de forma a possibilitar o desenvolvimento futuro de ações contextualizadas e efetivas. Para tanto, apresenta a seguinte questão de pesquisa: qual a prevalência do uso de substância psicoativa pelas gestantes e puérperas? Diante do exposto, o presente estudo objetiva investigar a prevalência do uso de substâncias psicoativas em gestantes e puérperas atendidas no ambulatório de um Hospital Escola.

\section{Método}

Trata-se de um estudo transversal, descritivo, desenvolvido em um ambulatório referência para acompanhamento de gestantes e puérperas de alto risco, localizado em um Hospital Escola do interior do Rio Grande do Sul, no período de agosto a novembro de 2018.

Para realização do cálculo amostral, utilizou-se o total de habitantes da cidade de Pelotas, o qual é de 328.275 , sendo que $1 \%$ corresponde ao número de mulheres grávidas, totalizando 3.283 gestantes. Para calcular o número de gestantes de alto risco, considerou-se os parâmetros 
5 | Lopes KB, Ribeiro JP, Dilélio AA, Tavares AR, Franchini B, Hartmann M

propostos na Portaria № 650, de 5 de outubro de 2011, em que 15\% de gestantes seriam identificadas como de alto risco, estimando-se 493 gestantes por ano. ${ }^{14}$ Empregou-se um erro amostral de $2 \%$, nível de confiança de $99 \%$ e considerando a prevalência de $28,2 \%$ de gestantes usuárias de substâncias psicoativas, lícitas e ilícitas.

Especificamente neste estudo, consideraram-se 4/12 meses, visto que o objetivo foi investigar a prevalência do uso de substâncias psicoativas em gestantes de alto risco e puérperas atendidas nos meses de agosto, setembro, outubro e novembro de 2019. Assim, totalizando uma amostra de 143 gestantes e puérperas, as quais foram acrescidos $20 \%$ considerando possíveis perdas ou recusas, sendo necessário 172 mulheres. Ressalta-se que no período investigado foi possível captar além do estimado, obtendo-se 174 participantes.

Os critérios de inclusão foram ser gestante de alto risco ou puérpera em acompanhamento no período de coleta dos dados; comunicar-se verbalmente por meio da língua portuguesa, por ser a língua nativa dos entrevistadores; autorizar a divulgação dos dados em meio científico. O critério de exclusão, a saber: gestante ou puérpera que não apresentasse condições clínicas de participar, como dificuldade de comunicação e dispneia.

As gestantes e puérperas foram captadas na sala de espera do Ambulatório. Mediante o aceite, foram direcionadas para uma sala do referido serviço que estava disponível no momento, de forma a proporcionar privacidade e sigilo. Nessa ocasião, leu-se o Termo de Consentimento Livre e Esclarecido (TCLE), se maior de 18 anos, ou o Termo de Assentimento Livre e Esclarecido (TALE), se menor de 18 anos, sendo solicitada aos seus responsáveis legais a assinatura do TCLE. Após explicitada a ciência e aceite em participar da pesquisa por meio da assinatura, procedeu-se à coleta de dados.

A coleta de dados se deu por meio de um questionário semiestruturado, com 16 questões, sendo 8 de múltipla escolha e 8 questões abertas, que visou captar informações referentes a aspectos sociodemográficos e gineco-obstétricos da amostra e, na sequência, instrumento 
padronizado ASSIST, desenvolvido pela Organização Mundial da Saúde para avaliação do consumo de substâncias psicoativas. ${ }^{15}$

O instrumento contém oito questões que oferecem informações sobre o uso de substâncias psicoativas na vida e nos últimos três meses; problemas relacionados ao uso de substâncias; risco atual ou futuros problemas decorrentes do uso; indícios de dependência; uso de drogas injetáveis. ${ }^{15}$ Cada questão apresenta respostas estruturadas que correspondem a um valor numérico, que ao final da entrevista são somados para obter um escore final do ASSIST.

Para a análise dos dados, utilizou-se a estatística descritiva com cálculo de porcentagem para variáveis categóricas e média para variáveis numéricas por meio de frequência simples. Os dados foram armazenados em planilhas do Microsoft Excel ${ }^{\circledR}$ e a análise realizada por meio da estatística descritiva, sendo utilizado o Software estatístico Stata 12.0.

O referido estudo respeitou a Resolução 466/12, 510/16 e 580/18 que aborda a pesquisa envolvendo seres humanos e foi submetido ao Comitê de Ética em Pesquisa na Área da Saúde, sendo aprovado pelo parecer № 2.843.605, em 24 de agosto e 2018, e Certificado de Apresentação para Apreciação Ética № 96034518.6.0000.5316.

\section{Resultados}

\section{Perfil sociodemográfico e gineco-obstétrico das gestantes e puérperas}

A amostra deste estudo foi composta por 174 mulheres, sendo $162(93,1 \%)$ gestantes e 12 $(6,9 \%)$ puérperas, as quais realizaram acompanhamento no ambulatório. Ressalta-se que nesse período não houve perdas por recusa ou não preenchimento dos instrumentos de coleta de dados. As idades variaram entre 13 e 44 anos, com predomínio de 20 a 29 anos (n=90; 51,7\%). Com relação ao estado civil, a maioria relatou ser casada ( $\mathrm{n}=96 ; 55,1 \%)$, não possuir atividade 
7 | Lopes KB, Ribeiro JP, Dilélio AA, Tavares AR, Franchini B, Hartmann M

laboral ( $\mathrm{n}=93 ; 53,4 \%)$, com escolaridade correspondente ao ensino médio incompleto ( $\mathrm{n}=57$; 32,7\%) (Tabela 1).

Tabela 1 - Perfil sociodemográfico das gestantes e puérperas atendidas em pré-natal de uma ambulatório do interior do Rio Grande do Sul (Ago/2018 a Nov/2018).

\begin{tabular}{lc}
\hline Variáveis & $\mathbf{N}(\%)$ \\
\hline Idade (anos) & $13(7,4)$ \\
$\leq 19$ & $90(51,7)$ \\
20 à 29 & $55(31,6)$ \\
30 à 39 & $16(9,2)$ \\
$\geq 40$ & \\
Estado Civil & $77(44,2)$ \\
Solteira & $96(55,1)$ \\
Casada & $1(0,5)$ \\
Divorciada & \\
Escolaridade & $31(17,8)$ \\
Analfabeto & $23(13,2)$ \\
Ens. Fundamental Incompleto & $31(17,8)$ \\
Ens. Fundamental Completo & $57(32,7)$ \\
Ens. Médio Incompleto & $18(10,3)$ \\
Ens. Médio Completo & $10(5,7)$ \\
Ens. Superior Incompleto & $3(1,7)$ \\
Ens. Superior Completo & $1(0,5)$ \\
Pós-graduação & \\
\hline
\end{tabular}

Com relação ao perfil gineco-obstétrico, $55(32 \%)$ mulheres relataram ter tido 1 ou 2 abortos prévios; o número de gestações variou de 0 a 9, com média de 2,55 gestações; o número de filhos variou de 0 a 8 . A idade gestacional variou de 7 a 41 semanas, com predomínio de $\leq 19$ a 29 semanas ( $\mathrm{n}=96 ; 59,2 \%)$, seguida de 30 a $39(\mathrm{n}=59 ; 33,9 \%)$ e $\geq 40(\mathrm{n}=2 ; 2,1 \%)$. Referente as consultas de pré-natal, 114 (65\%) das mulheres referiram menos de seis consultas de pré-natal e $60(34,4 \%)$ mais de seis consultas gestacionais.

Das $174(100 \%)$ entrevistadas, 91 (52\%) utilizaram outro serviço durante a gestação; 141 (81\%) apresentaram problemas associados à gestação, com predomínio de Diabetes Mellitus Gestacional (DMG) (n=60; 34,48\%), Doença Hipertensiva Específica da Gestação (DHEG) (n=39; 
Prevalência do uso de substâncias psicoativas em gestantes e puérperas $\mid 8$

22,4\%) e Síndromes Hemorrágicas (n=19; 10,9\%); 29 (17\%) foram internadas durante a gestação devido a algum problema associado à mesma. Com relação ao tipo de parto, seis (50\%) puérperas referiram ter parto vaginal e seis $(50 \%)$ parto cesárea.

\section{Uso de substâncias psicoativas pelas gestantes e puérperas}

O uso de substâncias psicoativas na vida foi referido por 141(81\%) gestantes e puérperas atendidas no ambulatório, sendo o álcool $(\mathrm{n}=134 ; 77 \%)$ a substância mais utilizada, seguido dos derivados do tabaco $(\mathrm{n}=75,43,1 \%)$. Além disso, $33(18,9 \%)$ entrevistadas referiram nunca ter utilizado nenhuma substância psicoativa.

No que se refere ao uso nos últimos 3 meses, $50(28,7 \%)$ gestantes e $2(1,1 \%)$ puérperas referiram ter utilizado alguma substância psicoativa. De forma geral, o álcool foi a substância com maior prevalência de uso $(n=33 ; 63,4 \%)$ pelas gestantes e puérperas, seguido pelo uso do tabaco $(n=28,53,8 \%)$ e da maconha $(n=2,3,85 \%)$. Destaca-se que $20(38,4 \%)$ entrevistadas referiram fazer uso diário de tabaco (Tabela 2).

Tabela 2 - Frequência de uso das substâncias psicoativas pelas gestantes e puérperas atendidas em um Ambulatório do interior do Rio Grande do Sul (Ago/2018 a Nov/2018) nos últimos três meses

Frequência de uso

\begin{tabular}{|c|c|c|c|c|c|c|}
\hline Substância* & $\begin{array}{l}\text { Nunca } \\
\text { N (\%) }\end{array}$ & $\begin{array}{l}1 \text { ou } 2 \\
\text { vezes } \\
N(\%)\end{array}$ & $\begin{array}{c}\text { Mensalmente } \\
\text { N (\%) }\end{array}$ & $\begin{array}{c}\text { Semanalmente } \\
\mathbf{N}(\%)\end{array}$ & $\begin{array}{l}\text { Diariamente } \\
\text { ou quase todos } \\
\text { os dias } \mathrm{N}(\%)\end{array}$ & $\begin{array}{l}\text { Total } \\
\text { N (\%) }\end{array}$ \\
\hline Derivado & $112(64,3)$ & $2(3,8)$ & $1(1,9)$ & $5(9,6)$ & $20(38,4)$ & $28(53,8)$ \\
\hline
\end{tabular}

do Tabaco

$\begin{array}{lllllll}\text { Álcool } & 107(61,4) & 21(40,3) & 8(15,3) & 4(7,6) & - & 33(63,3)\end{array}$

$\begin{array}{llllll}\text { Maconha } & 132(79,3) & 2(3,8) & - & - & -\end{array}$

*Cocaína/Crack, Anfetaminas/Êxtase, Inalantes, Hipnóticos/Sedativos, Alucinógenos, Opioides não foram consumidas nos últimos três meses. Houve gestantes e puérperas que informaram o uso concomitante de mais de uma substância ou não utilizar nenhuma das substâncias investigadas.

Entre as gestantes e puérperas que utilizaram alguma substância nos últimos 3 meses $(n=52 ; 29,8 \%), 26(50 \%)$ referiram desejo ou urgência para consumir derivados do tabaco, sendo 
9 | Lopes KB, Ribeiro JP, Dilélio AA, Tavares AR, Franchini B, Hartmann M

que $22(42,3 \%)$ manifestaram ter desejo diariamente ou quase todos os dias. Em relação às demais substâncias psicoativas, $16(30,7 \%)$ relataram desejo ou urgência de consumir álcool e 2 $(3,8 \%)$ de consumir maconha, nos últimos 3 meses (Tabela 3$)$.

Tabela 3 - Desejo ou urgência para consumir substâncias psicoativas nos últimos três meses, pelas gestantes e puérperas atendidas em um Ambulatório do interior do Rio Grande do Sul(Ago/2018 a Nov/2018)

\begin{tabular}{lccccc}
\hline & & & \multicolumn{2}{c}{ Desejo ou urgência } \\
& Nunca & 1 ou 2 vezes & Mensalmente & Semanalmente & Diariamente ou quase \\
& N (\%) & N (\%) & N (\%) & N (\%) & todos os dias \\
Substância* & & & & $3(1,7)$ & N (\%) \\
\hline $\begin{array}{l}\text { Derivados do } \\
\text { Tabaco }\end{array}$ & $30(17,2)$ & $1(0,5)$ & - & $12,6)$ \\
Álcool & $40(22,9)$ & $9(5,1)$ & $4(2,3)$ & $1(0,5)$ & $2(1,1)$ \\
Maconha & $54(31,0)$ & $1(0,5)$ & $1(0,5)$ & - & -
\end{tabular}

${ }^{*}$ Cocaína/Crack, Anfetaminas/Êxtase, Inalantes, Hipnóticos/Sedativos, Alucinógenos, Opioides não foram citadas quanto a desejo ou urgência de consumo. Houve gestantes e puérperas que informaram o uso concomitante de mais de uma substância ou não utilizar nenhuma das substâncias investigadas.

Das mulheres que utilizaram substâncias psicoativas nos últimos 3 meses ( $n=52,29,8 \%)$, oito $(15,3 \%)$ gestantes e puérperas assinalaram associação do uso de derivados do tabaco com problemas de saúde, social, legal ou financeiro em função do uso. Destaca-se que três $(5,7 \%)$ referiram deixar, alguma vez, de fazer algo que era normalmente esperado por elas devido ao uso de álcool e uma (1,9\%) devido ao uso de derivados do tabaco.

Entre as gestantes e puérperas que utilizaram alguma substância psicoativa na vida ( $\mathrm{n}=$ 141, 81,6\%), 14 (9,9\%) mencionaram tentar diminuir ou parar o uso de derivado de tabaco, $5(3,5 \%)$ de álcool e 1 (0,7\%) de maconha, nos últimos 3 meses. Dezoito (12,7\%) afirmaram que amigos, parentes ou outros familiares demonstraram preocupação com o uso de derivados de tabaco, sendo dez (7\%) devido ao seu uso de álcool e duas $(1,4 \%)$ devido ao uso de maconha.

Entre as $174(100 \%)$ gestantes e puérperas entrevistas nesta pesquisa, 130 (74,7\%) apresentaram baixo risco de dependência de substância psicoativa, sendo 127 (72,9\%) 
relacionadas ao álcool, $35(20,1 \%)$ de derivados de tabaco, $16(9,2 \%)$ a maconha, não havendo necessidade de qualquer intervenção.

Além disso, 38 (21,8\%) gestantes e puérperas entrevistadas apresentaram risco moderado de dependência, sendo $35(20,1 \%)$ relacionadas à dependência ao tabaco, $7(4,0 \%)$ ao álcool e 4 $(2,3 \%)$ à maconha. Fato que aponta a necessidade de intervenção breve a essas mulheres. Outras cinco $(2,8 \%)$ apresentaram risco grave de dependência ao tabaco, apresentando a necessidade de encaminhamento para tratamento intensivo (Tabela 4).

Tabela 4 - Risco de dependência das gestantes e puérperas, atendidas em um Ambulatório do interior do Rio Grande do Sul (Ago/2018 a Nov/2018), referente aos três últimos meses de vida

\begin{tabular}{lccc}
\hline & Baixo Risco & Risco Moderado & $\begin{array}{l}\text { Risco } \\
\text { Substância* }\end{array}$ \\
& N (\%) & N (\%) & N (\%) \\
\hline Derivados do Tabaco & $35(20,1)$ & $35(20,1)$ & $5(2,8)$ \\
Álcool & $127(72,9)$ & $7(4,0)$ & - \\
Maconha & $16(9,2)$ & $4(2,3)$ & - \\
Cocaína e Crack & $6(3,4)$ & - & - \\
Anfetaminas & $3(1,7)$ & - & - \\
Inalantes & $1(0,5)$ & - & - \\
Hipnóticos/Sedativos & $5(2,8)$ & - & - \\
Alucinógenos & $1(0,5)$ & - & - \\
Opioides & $1(0,5)$ & - & \\
\hline
\end{tabular}

${ }^{*}$ Houve gestantes e puérperas que apresentaram risco de dependência para mais de uma substância.

\section{Discussão}

As gestantes e puérperas participantes deste estudo possuíam idades entre 13 e 44 anos, a maioria casada, sem atividade laboral e ensino médio incompleto. Perfil semelhante foi encontrado em pesquisa que avaliou o risco relacionado ao consumo de drogas entre gestantes, em que a média de idade variou entre 14 e 40 anos, sendo a maioria casada ou em união estável, com mais de 8 anos de estudo. ${ }^{11}$ 
11 | Lopes KB, Ribeiro JP, Dilélio AA, Tavares AR, Franchini B, Hartmann M

Quanto ao perfil gineco-obstétrico, estudo que analisou o perfil epidemiológico das gestantes de alto risco da região central do Rio Grande do Sul apontou que a maior parte era multípara, estava no segundo trimestre de gestação, possuía de um a três filhos e sem histórico de abortos prévios. ${ }^{16}$ Dados estes que corroboram com os achados na presente pesquisa.

No que se refere aos problemas associados à gestação, houve predomínio de DMG, seguido pela DHEG e Síndromes Hemorrágicas. Pesquisa que traçou o perfil epidemiológico de gestantes de alto risco, atendidas no Instituto da Mulher, Secretaria de Saúde do município de Francisco Beltrão, no estado do Paraná, evidenciou que maioria das gestantes foi encaminhada ao serviço especializado devido à Hipertensão Arterial Sistêmica (HAS), isto é, mulheres com HAS crônica ou as que desenvolveram DHEG, tendo como desfecho a cesariana na maior parte dos casos. ${ }^{17}$

Já a pesquisa de uma coorte de gestantes cujos partos foram financiados pelo Sistema Único de Saúde (SUS), no município de São Paulo, com uma amostra de 55.404 mulheres, sendo 14,8\% gestantes de alto risco, indicou que 2.360 foram internadas durante a gestação por complicações obstétricas, como infecções, doenças hipertensivas, diabetes e hemorragias, que correspondem às causas mais frequentes de mortalidade materna no Brasil e no mundo e similares aos resultados do presente manuscrito. ${ }^{18-19}$ Além disso, por meio de estudo realizado com gestantes atendidas em unidades de saúde da rede SUS, no município do Rio de Janeiro, foi possível observar que as mulheres com internações prévias ao parto tiveram piores desfechos maternos, maior frequência de internação pós-parto e em UTI, maior mortalidade materna que as gestantes sem internação prévia ao parto..$^{20}$

Destaca-se que o acompanhamento pré-natal pode contribuir para desfechos favoráveis, uma vez que oportuniza a detecção precoce do uso de substâncias psicoativas e o tratamento oportuno dos problemas relacionados. Assim, tornando possível controlar fatores de risco que trazem complicações para a saúde da mãe e do bebê. ${ }^{21}$ 
No ambulatório em que foi realizado este estudo, o acompanhamento das gestantes de alto risco está organizado a partir do agendamento das consultas, considerando que as gestantes atendidas e vinculadas a esse serviço deverão ter garantidos os exames e procedimentos necessários, bem como os leitos para internação no momento do parto ou em situação de intercorrências. No entanto, mais da metade das gestantes e puérperas entrevistadas referiu ter utilizado outro serviço durante a gestação, o que expõem as dificuldades enfrentadas em função do deficit de leitos obstétricos no município e nas cidades que o circundam.

Ainda quanto ao acompanhamento pré-natal do presente estudo, revelou-se elevado número de mulheres $(65,5 \%)$ com menos de seis consultas. No entanto, não é possível afirmar que as mesmas estejam com consultas abaixo do preconizado (mínimo de seis consultas), visto que a maioria $(59,2 \%)$ se encontrava com idade gestacional hábil para atingi-lo.

Com relação ao uso de substâncias psicoativas na vida, 134 (77\%) gestantes e puérperas desta pesquisa relataram já ter utilizado álcool, $75(43,1 \%)$ derivados do tabaco, $20(11,4 \%)$ maconha e $6(3,4 \%)$ cocaína e crack. Resultados similares foram encontrados em uma pesquisa realizada no Paraná, em que 209 gestantes responderam ao instrumento ASSIST, apontando que $86,1 \%$ já tinham experimentado álcool, $35,4 \%$ o tabaco, $5,7 \%$ a maconha e $1,4 \%$ a cocaína. ${ }^{10}$

No que diz respeito ao uso de substâncias nos últimos três meses, o álcool foi a substância com maior prevalência de uso pelas gestantes e puérperas, seguido pelo uso do tabaco. Tais resultados vão ao encontro dos achados da National Survey on Drug Use and Health, a qual apontou que 4,7\% das mulheres grávidas usavam substâncias psicoativas, dentre estas, $13,6 \%$ utilizavam tabaco e 9,3\% álcool. ${ }^{21}$

Ressalta-se que os derivados do tabaco foram as substâncias psicoativas com maior frequência de uso pelas gestantes e puérperas do estudo, sendo relatada a sua utilização diária por $20(38,46 \%)$ entrevistadas. O tabaco também foi mencionado como a substância que desencadeia, com maior frequência, desejo ou urgência de consumo. Pesquisa realizada em 
13 | Lopes KB, Ribeiro JP, Dilélio AA, Tavares AR, Franchini B, Hartmann M

Maringá com 25 gestantes usuárias de drogas evidenciou que as mesmas conheciam os malefícios que o uso das drogas pode ocasionar durante a gravidez, entretanto possuíam dificuldade de abandonar o vício justamente pela dependência que estas ocasionam. ${ }^{11}$

Em função disso, muitas mulheres sentem medo e culpa decorrente da possibilidade de agravos ao feto. ${ }^{13}$ Consoante a esses achados, o presente estudo apontou que o tabaco foi a substância que obteve maior taxa de tentativas de diminuição ou abstinência, seguido pelo álcool e maconha, entre as gestantes e puérperas.

Entre as gestantes e puérperas que utilizaram substâncias psicoativas nos últimos três meses e assinalaram ter tido problemas de saúde, social, legal ou financeiro em função desse uso, três $(5,7 \%)$ referiram deixar, alguma vez, de fazer algo que era normalmente esperado por elas devido ao uso de álcool e uma (1,92\%) devido ao uso de derivados do tabaco. Pesquisadores apontam que o uso drogas pela figura materna pode acarretar problemas no âmbito familiar e social, tais como a desqualificação no mercado de trabalho em razão dos efeitos do uso, da descontinuidade no vínculo conjugal e do comprometimento no vínculo familiar e negligência com os filhos. ${ }^{22}$

O presente estudo expôs que amigos, parentes ou outros familiares demonstraram preocupação com maior frequência quando a substância em uso pela mulher era derivada do tabaco, seguida pelo uso de álcool e de maconha. Nesse contexto, destaca-se o potencial da rede social de apoio das gestantes usuárias de substâncias psicoativas para o cuidado da saúde mental, uma vez que, quando fortalecida, pode auxiliá-las no enfrentamento de sua condição, apoiando e incentivando a busca por ajuda. ${ }^{23}$

Com relação ao grau de dependência, a maioria das entrevistas $(74,7 \%)$ apresentou baixo risco de dependência à substância psicoativa, sem necessidade de qualquer intervenção. No entanto, o fato de gestantes e puérperas usuárias de substâncias psicoativas serem discriminadas devido à associação do uso com irresponsabilidade contribui para a negação do 
consumo e, também, para a não adesão à assistência pré-natal por vergonha, sentimento de medo e constrangimento. ${ }^{24}$

Por outro lado, cabe observar que um número expressivo de mulheres apresentou grau de dependência de moderado a grave $(24,7 \%)$, sendo a maioria dos casos relacionada ao uso de tabaco $(22,9 \%)$. Esses resultados corroboram com os achados de um estudo realizado em um hospital no interior do Rio grande do Sul, com uma amostra de 2.656 gestantes, em que 769 $(28,2 \%)$ apresentavam histórico de tabagismo atual ou prévio, e $49 \%$ dessas gestantes fumavam de 11 a 20 cigarros nos seis meses anteriores à gestação atual. ${ }^{25}$

Ressalta-se que o grau de dependência moderada sinaliza a necessidade de realização de uma intervenção breve e o grau de dependência grave exige que seja dispensado à usuária um tratamento intensivo. ${ }^{17}$ No entanto, apenas três entrevistadas mencionaram realizar tratamento.

Considerando que o uso de substâncias psicoativas está associado a piores desfechos maternos, perinatais e do desenvolvimento infantil, cabe aos profissionais de saúde o desenvolvimento de competências técnicas e psicossociais para apoiar e estimular as mulheres. Ressalta-se que o melhor momento para as abordar é o gestacional, visto que uma das principais motivações para a suspensão do uso é a preocupação com a gestação e a saúde do filho, além de ser um período em que é possível realizar uma intervenção adequada e eficaz, protegendo o binômio mãe e feto de desfechos perinatais desfavoráveis. ${ }^{26-27}$

Como limitações do estudo, os resultados ora apresentados não podem ser generalizados a outras populações, pois foi desenvolvido em um serviço específico que atente uma população com características próprias, visto ser um ambulatório referência para acompanhamento de gestantes e puérperas de alto risco.

\section{Conclusão}


15 | Lopes KB, Ribeiro JP, Dilélio AA, Tavares AR, Franchini B, Hartmann M

Ressalta-se que os derivados do tabaco se constituem nas substâncias com maior frequência de uso, apontadas como as que desencadeiam desejo ou urgência de consumo. Além, disso, foram as substâncias que apresentaram maior taxa de tentativas de diminuição ou abstinência, como também cujas gestantes e puérperas apresentaram grau de dependência de moderado a grave.

Os resultados do presente estudo apontam a pertinência de se avaliar o uso de substâncias psicoativas por gestantes e puérperas, uma vez que se trata de uma população singular cujo perfil sociodemográfico, somado ao perfil gineco-obstétrico, suscita maior atenção dos profissionais de saúde devido aos reflexos que impõe sobre a saúde da mãe e do bebê. Nesse sentido, o acolhimento do profissional, despido de preconceitos e estereótipos, constitui-se em uma ferramenta que potencializa a anamnese e, consequentemente, a identificação de fatores que coloquem em risco tanto a saúde física quanto a saúde mental dessas mulheres, tais como DMG, DHEG, síndromes hemorrágicas e o uso de substâncias psicoativas.

No que se refere especificamente ao rastreio e avaliação do uso de substâncias psicoativas, destaca-se que a utilização de instrumentos padronizados e validados como o ASSIST possui grande valor, especialmente no período gravídico-puerperal, quando a mulher está mais sensibilizada em relação aos efeitos nocivos que as substâncias podem acarretar ao seu filho. Além disso, o ASSIST é um instrumento de fácil compreensão, rápida aplicação, que explora os diferentes tipos de substâncias e direciona o profissional ao tipo de intervenção necessária de acordo com o grau de dependência apontado.

Esse panorama expõe que o uso de tabaco na gestação e puerpério constitui-se em um desafio à saúde pública e aos profissionais de saúde, suscitando o desenvolvimento de ações educativas que exponham as consequências do uso de substância, bem como a captação precoce dessas mulheres para a elaboração de plano terapêutico singular. Esse plano precisa englobar estratégias de redução e/ou abstinência aos derivados do tabaco, considerando o grau de dependência desenvolvido, a sua singularidade e o período de vida vivenciado. Além disso, 
salienta-se a importância da rede de apoio nesse processo, visto como uma fonte de fortalecimento, podendo auxiliar no enfrentamento, de modo a apoiar e incentivar a cessar o uso.

A partir de tais ações, ampliam-se as potencialidades do acompanhamento desenvolvido por meio das consultas de pré-natal, puerpério e puericultura, no que tange à redução de complicações maternas e fetais. Aponta-se a necessidade de que estudos futuros investiguem a assistência ofertada às gestantes e puérperas usuárias de substâncias psicoativas, identificando as estratégias de captação e cuidado.

\section{Referências}

1. Substance Abuse and Mental Health Services Administration (SAMHSA). Key substance use and mental health indicators in the United States: Results from the 2018 National Survey on Drug Use and Health [Internet]. Rockville (MD): SAMHSA; 2019 [cited 2020 Sept 02]. Available from: https://www.samhsa.gov/data/sites/default/files/cbhsqreports/NSDUHNationalFindingsReport2018/NSDUHNationalFindingsReport2018.pdf

2. Bastos FI, Bertoni N, organizadores. Pesquisa Nacional sobre o uso de crack: quem são os usuários de crack e/ou similares do Brasil? quantos são nas capitais brasileiras? [Internet]. Rio de Janeiro: ICICT/FIOCRUZ; 2014 [acesso em 2020 set 02]. Disponível em: https://www.arca.fiocruz.br/bitstream/icict/10019/2/UsoDeCrack.pdf

3. Rocha PC, Alves MTSSB, Chagas DC, Silva AAM, Batista RFL, Silva RA. Prevalência e fatores associados ao uso de drogas ilícitas em gestantes da coorte BRISA. Cad Saúde Pública. 2016; 32(1):e00192714. doi: 10.1590/0102-311X00192714

4. Pereira CM. Avaliação do uso de drogas na gestação e no período pós-parto [dissertação]. Universidade Estadual de Campinas, Faculdade de Ciências Médicas. Campinas: Universidade Estadual de Campinas; 2017 [acesso em 2020 set 02]. Disponível em: http://repositorio.unicamp.br/bitstream/REPOSIP/325572/1/Pereira_CynaraMaria_M.pdf

5. Tacon FSA, Amaral WN, Tacon KCB. Drogas ilícitas e gravidez: Influência na morfologia fetal. Femina [Internet]. 2018 [acesso em 2020 set 02];46(1):10-8. Disponível em: https://www.febrasgo.org.br/media/k2/attachments/VolZ46Z-Zn1-Z2018.pdf

6. Ferreira BRM, Miranda JKS. As complicações causadas pelo consumo de drogas lícitas e ilícitas 
17 | Lopes KB, Ribeiro JP, Dilélio AA, Tavares AR, Franchini B, Hartmann M

durante a gestação: um desafio para a equipe de enfermagem. Rev Recien. 2018;6(18):36-46. doi: 10.24276/rrecien2358-3088.2016.6.18.36-43

7. Aghamohammadi A, Zafari M. Crack abuse during pregnancy: maternal, fetal and neonatal complication. J Matern Fetal Neonatal Med. 2015;29(5):795-7. doi: 10.3109/14767058.2015.1018821

8. Santos RS, Estefanio MP, Figueiredo RM. Prevenção da síndrome alcoólica fetal: subsídios para a prática de enfermeiras obstétricas. Rev Enferm UERJ. 2017;25:e27793. doi: 10.12957/reuerj.2017.27793

9. Guimarães VA, Fernandes KS, Lucchese R, Vera I, Martins BCT, Amorim TA, et al. Prevalência e fatores associados ao uso de álcool durante a gestação em uma maternidade de Goiás, Brasil Central. Ciênc Saúde Colet. 2018;23(10):3413-20. doi: 10.1590/1413-812320182310.24582016

10. Santos RM, Gavioli A. Risco relacionado ao consumo de drogas de abuso em gestantes. Rev Rene. 2017;18(1):35-42. doi: 10.15253/2175-6783.2017000100006

11. Kassada DS, Marcon SS, Waidman MAP. Percepções e práticas de gestantes atendidas na atenção primária frente ao uso de drogas. Esc Anna Nery Rev Enferm. 2014;18(3):428-34. doi: 10.5935/1414-8145.20140061

12. Botelho APM, Rocha RC, Melo VH. Uso e dependência de cocaína/crack na gestação, parto e puerpério. Femina [Internet]. 2013 [acesso em 2019 jul 29];41(1):23-32. Disponível em: http://files.bvs.br/upload/S/0100-7254/2013/v41n1/a3777.pdf

13. Lopes KB, Ribeiro JP, Porto AR. Care strategies for pregnant and puerperal psychoactive substances users: an integrative review. Rev Enferm UERJ. 2020;28:e49518. doi: 10.12957/reuerj.2020.49518

14. Ministério da Saúde (BR), Secretária da Atenção à Saúde. Portaria № 650, de 5 de outubro de 2011. Dispor sobre os Planos de Ação regional e municipal da Rede Cegonha, que são os documentos orientadores para a execução das fases de implementação da rede, assim como para o repasse dos recursos, o monitoramento e a avaliação da implementação da Rede Cegonha. Brasília (DF): Ministério da Saúde; 2011.

15. Ministério da Justiça e Cidadania, Secretaria Nacional de Políticas sobre Drogas. Guia do estudante: SUPERA: sistema para detecção do uso abusivo e dependência de substâncias psicoativas: encaminhamento, intervenção breve, reinserção social e acompanhamento [Internet]. Brasília (DF): Ministério da Justiça e Cidadania; 2017 [acesso em 2020 set 02]. Disponível em: https://www.supera.org.br/@/material/mtd/pdf/SUP/SUP_Mod3.pdf

16. Jantsch PF, Carreno I, Pozzobon A, Adami FS, Leal CS, Mathias TCS, et al. Principais características das gestantes de alto risco da região central do Rio Grande do Sul. Destaques Acadêmicos. 2017;9(3):27282. doi: 10.22410/issn.2176-3070.v9i3a2017.1534 
17. Costa LD, Cura CC, Perondi AR, França VF, Bortoloti DS. Perfil Epidemiológico de gestantes de alto risco. Cogitare Enferm [Internet]. 2016 [cited 2019 Jul 30];21(2):01-8. Available from: https://revistas.ufpr.br/cogitare/article/view/44192/28239

18. Moura BLA, Alencar GP, Silva ZP, Almeida MF. Internações por complicações obstétricas na gestação e desfechos maternos e perinatais, em uma coorte de gestantes no Sistema Único de Saúde no Município de São Paulo, Brasil. Cad Saúde Pública. 2018;34(1):e00188016. doi: 10.1590/0102-311X00188016

19. Say L, Berreix M, Chou D, Tunçalp Ö, Cottler S, McCaw-Binns A, et al. Maternal morbidity measurement tool pilot: study protocol. Reprod Health. 2016;69(13):01-6. doi: 10.1186/s12978-016-0164-6

20. Domingues RMSM, Hartz ZMA, Dias MAB, Leal MC. Avaliação da adequação da assistência prénatal na rede SUS do Município do Rio de Janeiro, Brasil. Cad Saúde Pública. 2012;28(3):425-37. doi: 10.1590/S0102-311X2012000300003

21. Coleman-Cowger V, Schauer G, Peters E. Marijuana and tobacco co-use among a nationally representative sample of US pregnant and non-pregnant women: 2005-2014 National Survey on Drug Use and Health findings. Drug Alcohol Depend. 2017;177(1):130-5. doi: 10.1016/j.drugalcdep.2017.03.025

22. Reis LM, Sales CA, Oliveira MLF. Narrativa de filha de usuária de drogas: repercussões no cotidiano familiar. Esc Anna Nery Rev Enferm. 2017;21(3):e20170080. doi: 10.1590/2177-9465-EAN-2017-0080

23. Wronski JL, Pavelski T, Guimarães AN, Zanotelli SS, Schneider JF, Bonilha ALL. Uso do crack na gestação: vivências de mulheres usuárias. Rev Enferm UFPE On Line [Internet]. 2016 [cited 2019 Jul 30];10(4):1231-39. Available from: https://periodicos.ufpe.br/revistas/revistaenfermagem/article/viewFile/11108/12577

24. Bardi ND, Antunes B, Roos CM, Olschowsky A, Pinho LB. Estigma e preconceito: vivência dos usuários de crack. Rev Latinoam Enferm. 2016;24:e2680. doi: 10.1590/1518-8345.0852.2680

25. Dias-Damé JL, Lindsay AC, Cesar JÁ. Cessação do tabagismo na gestação: estudo de base populacional. Rev Saúde Pública. 2018;53(3):1-11. doi: 10.11606/S1518-8787.2019053000619

26. Pereira C, Pacgnella RC, Parpinelli MA, Andreucci CB, Zanardi DM, Souza R, et al. Drug use during Pregnancy and its consequences: a nested case control study on severe maternal morbidity. Rev Bras Ginecol Obstet. 2018;40(9):518-26. doi: 10.1055/s-0038-1667291

27. Tavares AR, Ribeiro JP, Porto AR, Lopes KB, Hartmann M, Leon ER, et al. Perfil das gestantes atendidas em um ambulatório no Rio Grande do Sul e o uso de substâncias psicoativas. Rev Eletrônica Acervo Saúde. 2020;13(1):e5848. doi: 10.25248/reas.e5848.2021 
19 | Lopes KB, Ribeiro JP, Dilélio AA, Tavares AR, Franchini B, Hartmann M

Editora Científica: Tânia Solange Bosi de Souza Magnago

Editora associada: Graciela Dutra Sehnem

\author{
Autor correspondente \\ Juliane Portella Ribeiro \\ E-mail: ju_ribeiro1985@hotmail.com \\ Endereço: Rua Gomes Carneiro, 01 - Balsa, Pelotas - RS. CEP: 96010-610
}

Financiamento/Fomento: Fundação de Amparo à Pesquisa do Estado do Rio Grande do Sul (FAPERGS) com a bolsa Iniciação Cientifica - Edital CPESQ № 003/2019.

\title{
Contribuições de Autoria
}

1 - Karen Barcelos Lopes

Contribuiu na concepção, projeto, análise e interpretação dos dados do manuscrito.

\section{2 - Juliane Portella Ribeiro}

Contribuições: Contribuiu na concepção, projeto, análise e interpretação dos dados e aprovação final da versão a ser publicada.

\section{3 - Alitéia Santiago Dilélio}

Contribuiu com a análise e interpretação dos dados.

\section{4 - Amanda do Rosário Tavares}

Contribuiu com a análise e interpretação dos dados.

\section{5 - Beatriz Franchini}

Contribuiu com a revisão final.

\section{6 - Melissa Hartmann}

Contribuiu com a análise e interpretação dos dados.

\section{Como citar este artigo}

Lopes KB, Ribeiro JP, Dilélio AS, Tavares AR, Franchini B, Hartmann M. Prevalência do uso de substâncias psicoativas em gestantes e puérperas. Rev. Enferm. UFSM. 2021 [Acesso em: Ano Mês Dia]; vol.11 e45: 1-19. DOI: https://doi.org/10.5902/2179769254544 